\title{
Stress-mediated p38 activation promotes somatic cell reprogramming
}

\author{
Xinxiu Xu ${ }^{1,2}$, Quan Wang ${ }^{2}$, Yuan Long ${ }^{2}$, Ru Zhang ${ }^{1}$, Xiaoyuan Wei ${ }^{1}$, Mingzhe Xing ${ }^{1}$, Haifeng Gu ${ }^{2}$ Xin Xie ${ }^{1,2}$ \\ ${ }^{1}$ Laboratory of Receptor-based Bio-medicine, Shanghai Key Laboratory of Signaling and Disease Research, School of Life Sci- \\ ences and Technology, Tongji University, Shanghai 200092, China: ${ }^{2}$ Stake Key Laboratory of Drug Research, the National Center \\ for Drug Screening, Shanghai Institute of Materia Medica, Chinese Academy of Sciences, Shanghai 201203, China
}

Environmental stress-mediated adaptation plays essential roles in the evolution of life. Cellular adaptation mechanisms usually involve the regulation of chromatin structure, transcription, mRNA stability and translation, which eventually lead to efficient changes in gene expression. Global epigenetic change is also involved in the reprogramming of somatic cells into induced pluripotent stem (iPS) cells by defined factors. Here we report that environmental stress such as hyperosmosis not only facilitates four factor-mediated reprogramming, but also enhances two or one factor-induced iPS cell generation. Hyperosmosis-induced p38 activation plays a critical role in this process. Constitutive active p38 mimics the positive effect of hyperosmosis, while dominant negative p38 and p38 inhibitor block the effect of hyperosmosis. Further study indicates stress-mediated p38 activation may promote reprogramming by reducing the global DNA methylation level and enhancing the expression of pluripotency genes. Our results demonstrate how simple environmental stress like hyperosmosis helps to alter the fate of cells via intracellular signaling and epigenetic modulation.

Keywords: induced pluripotent stem cells; iPS; hyperosmosis; $\mathrm{NaCl}$; stress; $\mathrm{p} 38$; reprogramming Cell Research (2013) 23:131-141. doi:10.1038/cr.2012.143; published online 9 October 2012

\section{Introduction}

Environmental stress has long been associated with numerous human diseases. Nevertheless, not all stress is harmful. Instead, environmental stress below the mutation threshold might play an essential role in the adaptation and evolution of life [1]. Classical responses of animals to stress, such as the 'fight-or-flight response' or 'general adaptation syndrome', are controlled by hormones at the organismal level [2]. At the cellular level, the adaptation mechanisms usually involve efficient changes in the gene expression programs mediated by intracellular signaling networks. Regulation of chromatin structure, transcription, mRNA stability and translation are some of the critical steps for cells to achieve generic and specific responses to different stresses [3].

Somatic cells can be reprogrammed into induced

Correspondence: Xin Xie

Tel: +86-21-50801313 ex 156

E-mail: xxie@mail.shcnc.ac.cn

Received 30 March 2012; revised 26 July 2012; accepted 17 August 2012; published online 9 October 2012 pluripotent stem (iPS) cells by ectopic expression of defined factors [4]. The exact underlying mechanisms remain unclear, but a global epigenetic change is believed to be involved in iPS induction [5]. To avoid the use of oncogenes and incorporation of viral DNA sequences, and to make iPS cells more amenable for therapeutic application, many efforts have been taken to modify the original protocol by using reduced number of factors [6], non-integrating gene delivery approaches [7], or cell permeable proteins to trigger the reprogramming [8]. A number of small molecules, especially those affecting epigenetic modulations, such as VPA, butyrate, BIX01294 and 5-Aza-2'-deoxycytidine (5-Aza), have also been reported to facilitate iPS cell induction [9].

To identify better small molecule enhancers of reprogramming, we developed a screening system with 96-well plates. Unexpectedly, we found that iPS cells appeared earlier and the induction efficiency was higher in the corner wells than the center ones. After ruling out the possible effect of $\mathrm{pH}$ and nutritional changes, we discovered that it was the increased osmotic pressure in the corner wells that facilitated iPS cell generation. Hyperosmosis not only facilitated four factor-mediated repro- 
gramming, but also enhanced two or one factor-induced iPS cell generation in combination with other chemicals. Hyperosmosis has been reported to activate many stressrelated signaling pathways, including the MAPK pathways [10]. Our study indicated that hyperosmosis-induced p38 activation played a critical role in facilitating reprogramming. The constitutive active p38 mimicked the positive effect of hyperosmosis, while dominant negative p38 and p38 inhibitor PD169316 blocked the effect of hyperosmosis. Further study indicates stress-mediated p38 activation might promote reprogramming by reducing the global DNA methylation level and enhancing the expression of pluripotency genes. We thus demonstrate how simple environmental stress like hyperosmosis could help to alter the fate of cells via intracellular signaling and epigenetic modulation.

\section{Results}

Hyperosmosis promotes four factor-mediated reprogramming in mouse embryonic fibroblasts (MEFs)

We established a 96-well-plate-based chemical screening system for four-factor (4F)-induced reprogramming in OG2 MEFs which carry a transgenic Oct4 promoter driven-GFP reporter [11] (Supplementary information, Figure S1A). We unexpectedly observed that the reprogramming efficiency at the corner wells were higher than the center ones. Corner or edge effects are generally associated with increased osmotic pressure and $\mathrm{pH}$. By using $\mathrm{NaCl}$ and $\mathrm{NaHCO}_{3}$ to mimic the osmotic and $\mathrm{pH}$ changes, we found only the addition of $\mathrm{NaCl}(50$ $100 \mathrm{mM}$ ) significantly increased the number of $\mathrm{GFP}^{+}$ colonies. Change of $\mathrm{pH}$ or the percent of serum did not affect iPS cell generation (Supplementary information, Figure S1B). More careful analyses indicated $\mathrm{NaCl}$ supplementation not only increased the number of $\mathrm{GFP}^{+}$ colonies, but also shortened the reprogramming process. At day 8 , more than $10 \mathrm{GFP}^{+}$colonies could be observed in NaCl-added wells (5 $000 \mathrm{MEFs} /$ well), while the control wells had almost none (Figure 1A). The non-ionic osmotic pressure regulator, sucrose $(200 \mathrm{mM})$ [12], also increased the reprogramming efficiency by $\sim 10$-fold in 4F-transduced MEFs (Figure 1B). At day 14, FACS data revealed a remarkable enhancement of reprogramming efficiency with $>20 \%$ cells being $\mathrm{GFP}^{+}$(Figure 1C).

Hyperosmosis is effective when implemented at the early stage of reprogramming and the effect is additive to other chemicals

To clarify whether hyperosmosis facilitates the reprogramming process or promotes the self-renewal of iPS cells after reprogramming, we treated the $4 \mathrm{~F}$-transduced
MEF cells with $100 \mathrm{mM} \mathrm{NaCl}$ for $72 \mathrm{~h}$ starting on day 3, 6, or 9 . Starting $\mathrm{NaCl}$ treatment on day 9 had no beneficial effect, instead it slightly reduced the number of $\mathrm{GFP}^{+}$ colonies. In contrast, there was a statistically significant 8 - and 6-fold increase in the number of $\mathrm{GFP}^{+}$colonies in the cultures treated with $\mathrm{NaCl}$ starting on day 3 and 6 , respectively (Figure 1D and Supplementary information, Figure S1C). We also treated the 4F-transduced MEF cells with $50 \mathrm{mM}$ or $100 \mathrm{mM} \mathrm{NaCl}$ for various durations starting from day 3 . The early stage of reprogramming (day 3-8) benefited most from the hyperosmotic stress, since prolonged elevation of osmotic pressure did not further increase the reprogramming efficiency (Figure 1E). In fact, prolonged hyperosmotic stress (treatment beyond day 10) caused reduction in colony size and eventual reduction in colony number, indicating a cytotoxic effect. So the treatment duration for most of the later experiments was day 3-8. On fully reprogrammed iPS clones, hyperosmosis reduced the $\mathrm{GFP}^{+}$area and total GFP intensity, indicating the condition was harmful to iPS cells (Supplementary information, Figure S1D and S1E). MTT assay also revealed reduced viability in iPS cells when cultured in hyperosmotic condition (Supplementary information, Figure S1F). These data suggest that hyperosmosis promotes the generation of iPS colonies by facilitating the reprogramming process rather than enhancing the proliferation or self-renewal of iPS cells.

Next we tested $\mathrm{NaCl}$ in combination with two reported reprogramming enhancers, VPA and $\mathrm{LiCl}$ [13-14]. The combination of $\mathrm{NaCl}$ and VPA at low doses displayed a synergistic effect in enhancing reprogramming, while the combination of high doses did not even show additive effect. Similar to $\mathrm{NaCl}$, VPA also reduced the GFP area, total GFP intensity and cell viability in fully reprogrammed iPS cells (Supplementary information, Figure S2C-S2E). The combination of VPA and $\mathrm{NaCl}$ at high doses displayed higher toxicity towards iPS cells, which might compromise their effects in enhancing reprogramming. Similar synergistic effect between low dose of $\mathrm{NaCl}$ and low dose of $\mathrm{LiCl}$ was also observed (Supplementary information, Figure S2B).

Hyperosmosis facilitates the generation of mouse iPS cells with two or one factors and the iPS cells generated in such conditions are pluripotent

Hyperosmosis also facilitated reprogramming mediated by reduced number of factors and chemicals (Figure 1F). In OK, CHIR99021 and parnate-mediated reprogramming, $\mathrm{NaCl}(50 \mathrm{mM})$ led to a 5 -fold increase in the number of $\mathrm{GFP}^{+}$colonies at day 14. In OS, CHIR99021, Repsox and parnate-mediated reprogramming, hyperos- 



Figure 1 Hyperosmosis promotes iPS cell generation from MEFs. (A) Representative image and statistical data of the dose and time effect of $\mathrm{NaCl}$ on iPS $\left(\mathrm{GFP}^{+}\right.$) colony generation from 5000 4F-infected MEFs. Scale bar, $7 \mathrm{~mm}$. (B) The effect of $\mathrm{NaCl}(100 \mathrm{mM})$ or sucrose $(200 \mathrm{mM})$ in iPS cell induction in 4F-infected MEFs. (C) Representative FACS plots and statistical data of $\mathrm{GFP}^{+}$cell percentage at day 14 from 4F-infected MEFs treated with $\mathrm{NaCl}(100 \mathrm{mM})$ or sucrose (200 mM). (D) NaCl (100 $\mathrm{mM}$ ) was added for 3 days starting from day 3, 6 and 9 in 4F-infected MEFs and GFP ${ }^{+}$clonies were counted at day 14. Mean \pm SEM of a representative experiment, $n=3$. (E) 4F-infected MEFs were treated with $\mathrm{NaCl}$ for various durations starting from day 3 , and $\mathrm{GFP}^{+}$colonies were counted at day 14 . Mean \pm SEM of three independent experiments. (F) MEFs infected with $2 \mathrm{~F}$ or $1 \mathrm{~F}$ were treated with a combination of small molecules including $0.5 \mathrm{mM}$ VPA (V), $3 \mu \mathrm{M}$ CHIR99021 (C), $1 \mu \mathrm{M}$ RepSox (R) and $2 \mu \mathrm{M}$ parnate $(\mathrm{P})$ supplemented with $50 \mathrm{mM} \mathrm{NaCl}$ or not. Number of GFP ${ }^{+}$colonies was counted. Mean $\pm \mathrm{SEM}$ of three independent experiments. ${ }^{*} P<0.05,{ }^{* *} P<0.01,{ }^{* *} P<0.001$, versus control.

motic condition further improved the efficiency by 4 -fold at day 18. And the effect of the chemical combination (VPA, CHIR99021, parnate and Repsox) that successfully facilitated the one factor (Oct4)-induced reprogramming was further enhanced by 3 -fold in the hyperosmotic condition when examined at day 20 (Figure 1F).

PCR of genomic DNA of $2 \mathrm{~F}$ (OK or OS)- or $1 \mathrm{~F}(\mathrm{O})$ iPS clones confirmed the integration of the indicated but not the other retroviral factors (Supplementary information, Figure S3A and S3B). Real-time PCR analysis 
confirmed the reactivation of the endogenous Oct4, Sox2, Nanog and Rexl and the silencing of viral genes in these iPS clones (Supplementary information, Figure S3C and S3D). These iPS cells maintain GFP expression and ESlike morphology, and express typical pluripotency markers, such as alkaline phosphatase, SSEA1 and Nanog (Figure 2A). Bisulfite genomic sequencing analyses of the Oct4 and Nanog promoters revealed that both were demethylated in iPS cells, similar to mES cells and in contrast to the parental MEFs (Figure 2B). Subcutaneous injection of the iPS cells into NOD-SCID mice led to teratoma formation, with tissues derived from all three germ layers (Figure 2C). We obtained adult chimaeras from all three clones (OK-1, OS-1 and O-1) as determined by coat color (Figure 2D and Supplementary information, Table S3). One of the male chimaeras from clone OK-1 was crossed with ICR females and 7 of the total $47 \mathrm{~F} 1$ mice showed agouti coat color (Figure 2E and Supplementary information, Table S3), confirming germline transmission.

Hyperosmosis promotes iPS cell generation by activating the p38 pathway

Hyperosmosis was reported to activate many stressrelated signaling pathways [10]. We found hyperosmosis significantly activated JNK and p38, two major regulators of stress responses, and to a lesser extent, the ERK pathway (Figure 3A and 3B). Blocking JNK and ERK pathways with SP600125 and PD98059 further enhanced the positive effect of hyperosmosis on reprogramming (Figure 3C). The result agrees with previous reports that the activation of ERK and JNK pathways was detrimental to iPS cell induction [15]. Interestingly, blocking hyperosmosis induced-p38 activation with PD169316 totally abolished its promoting effect on reprogramming (Figure 3C). Anisomycin and TNF- $\alpha$, two well known activators of $\mathrm{p} 38$ and its downstream target MAPKAPK2 (MK2) (Supplementary information, Figure S4A, S4B and S4D), also enhanced iPS cell generation and such enhancement was blocked by PD169316 (Figure 3D). Sucrose also activated the p38 pathway in MEF cells (Supplementary information, Figure S4C). In contrast, VPA was believed to enhance reprogramming via HDAC inhibition. It did not induce p38 activation and its effect was not blocked by PD169316 (Supplementary information, Figure S4E and S4F).

To confirm the role of $\mathrm{p} 38$ in reprogramming, we constructed the constitutively active (CA)-p38, which contains D176A and F327S mutations [16], and the dominant negative (DN)-p38 with T180A and Y182F mutations [17]. Western blot analysis confirmed the expression and correct function of these constructs (Sup- plementary information, Figure S5). When expressed in MEFs, only the CA-p38 promoted 4F-driven iPS cell generation in standard iso-osmotic medium. WT-p38 and DN-p38 had no effect. However, in hyperosmotic medium, DN-p38 totally blocked hyperosmosis-enhanced reprogramming (Figure 3E). Similar to $\mathrm{NaCl}$ treatment, the CA-p38 was only beneficial at the reprogramming stage. If CA-p38 was blocked with PD169316 at early reprogramming stage (before day 9), no enhancement was observed (Figure 3F). But if PD169316 was added after day 15 , prolonged activation of $\mathrm{p} 38$ also caused reduction in iPS colonies (Figure 3F). The time courses of $\mathrm{NaCl}$ and CA-p38 on reprogramming were slightly different, which was probably due to the variable degrees of p38 activation.

Hyperosmotic stress-mediated p38 activation may promote reprogramming by reducing the global DNA methylation level

Endogenous expression of Nanog, Oct4 and Sox2 is essential for successful reprogramming of somatic cells. During the 4F-mediated reprogramming, hyperosmotic stress induced a significant increase in Nanog, Oct4 and Sox 2 mRNA transcription (Figure 4A). By using luciferase reporters driven by the promoters of Nanog, Oct4 or Sox2, we found hyperosmosis significantly activated all these promoters without the presence of Yamanaka factors (Figure 4B). Interestingly, p38 inhibitor PD169316 $(10 \mu \mathrm{M})$ only blocked the activation of Nanog and Sox 2 reporters, but not the Oct4 reporter (Figure $4 \mathrm{~B})$, indicating the involvement of other pathways in the activation of Oct4 transcription by hyperosmosis. DNA methylation levels at the loci of Oct4 and Nanog promoters after $\mathrm{NaCl}$ treatment were determined by bisulfite sequencing. As shown in Figure 4C, 4D and Supplementary information, Figure S6, more than $60 \%$ of the CpGs in the promoter regions of Oct4 and Nanog were methylated in MEFs. In contrast, ES cells had almost no methylation in the promoter regions of these genes. $\mathrm{NaCl}$ treatment significantly reduced the level of methylation in both Oct4 and Nanog promoters, supporting that hyperosmotic conditions can facilitate the activation of these pluripotency genes.

Hyperosmotic stress has been reported to activate many genes critical for cell differentiation, including Keratin K1 and K10 for epidermal differentiation, and MEF2 for skeletal muscle differentiation [18-19]. P38 activation has also been found to induce cell differentiation [20]. The fact that hyperosmosis and p38 activation facilitate both differentiation and reprogramming led us to speculate that stress might affect gene transcription at a global level. Using dot blot analysis, we found 
A
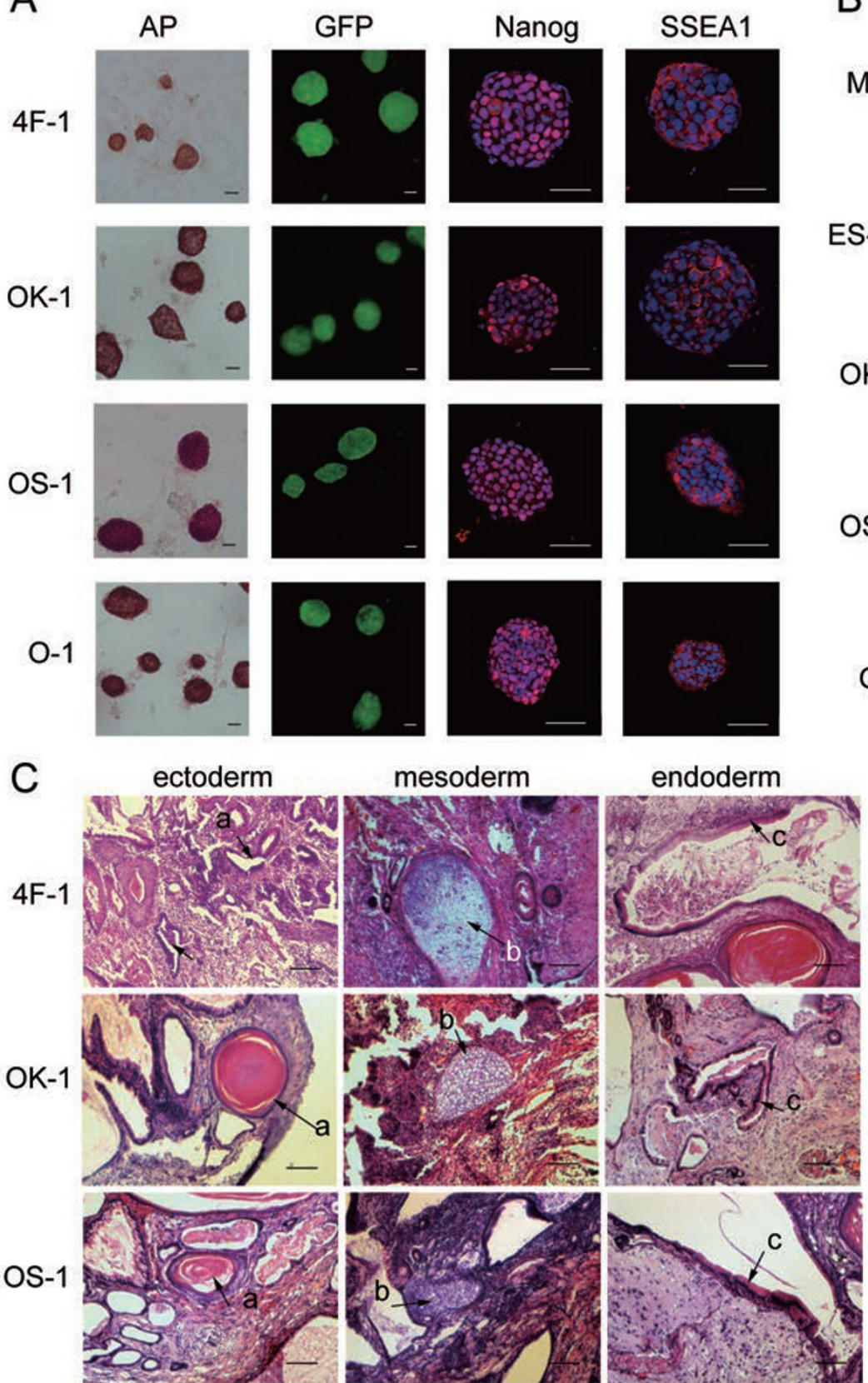

OS-1
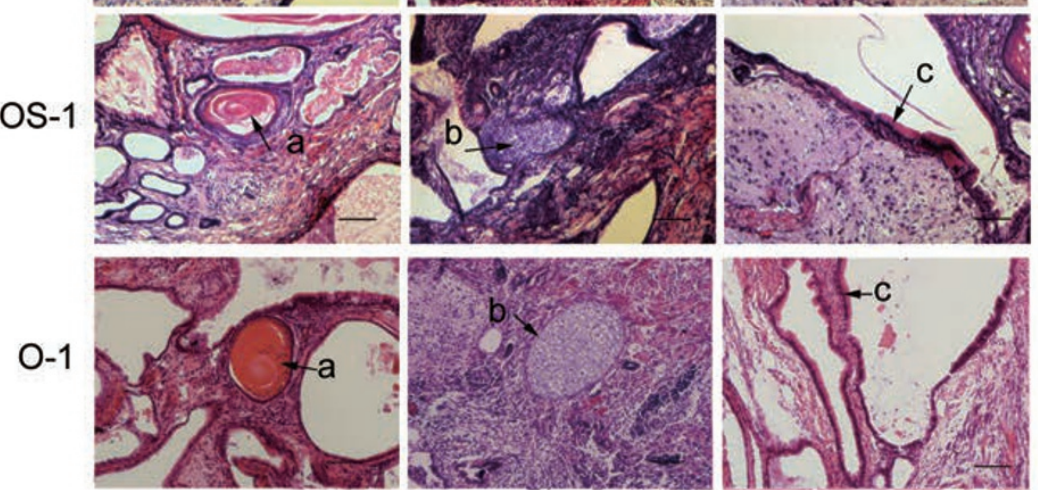

B

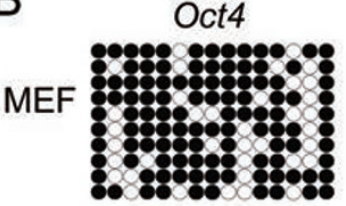

ES-14


OS-1

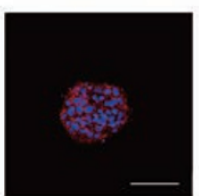

0-1
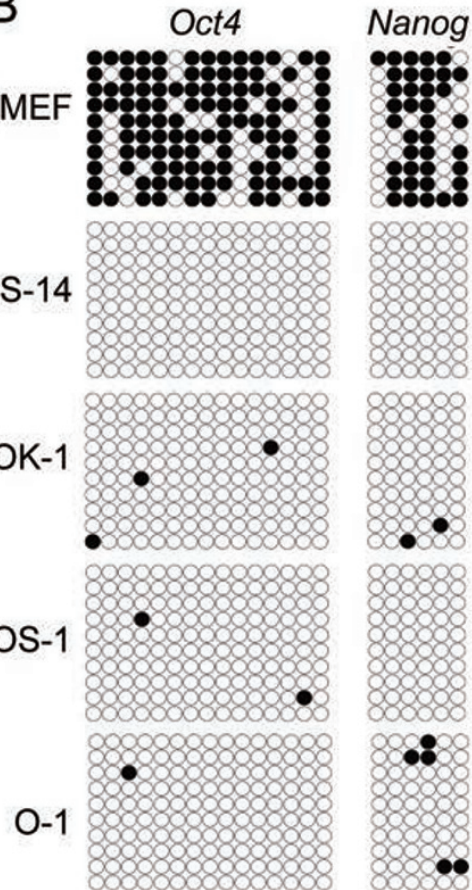

OK-1
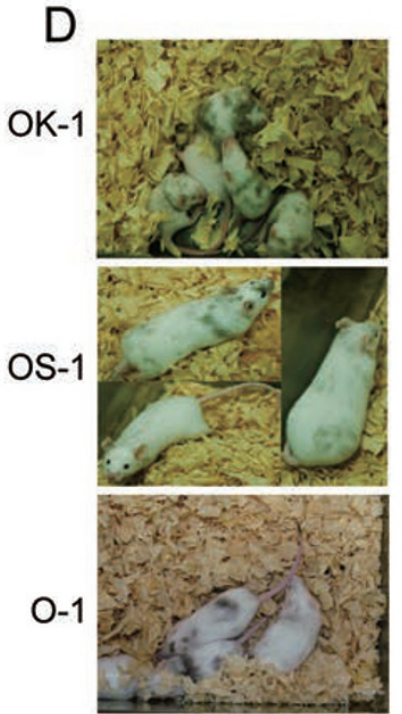

E

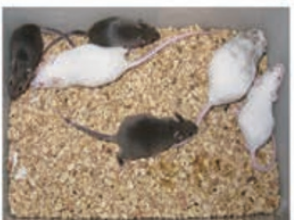

Figure 2 Pluripotency of iPS cells generated in hyperosmotic conditions. (A) Alkaline phosphatase staining, GFP expression and immunofluorescent staining of pluripotency marker SSEA-1 and Nanog in 4F-, 2F (OK or OS)- and 1F (O)-iPS clones. Scale bar, $50 \mu \mathrm{m}$. (B) DNA methylation analysis of the Oct4 and Nanog promoters by bisulfite genomic sequencing in ES-14 and iPS clones. Open and closed circles indicate unmethylated and methylated CpGs, respectively. (C) Hematoxylin and eosin-stained sections of teratomas formed with iPS clones. Typical structures of the three embryonic germ layers were pointed out by arrows: a, epidermis (ectoderm), b, cartilage (mesoderm) and c, glandular epithelium (endoderm). Scale bar, $50 \mu \mathrm{m}$. (D) Chimeric mice produced with 2F- and 1F-iPS clones. (E) A chimeric mouse generated with iPS clone OK-1 and its agouticoat-colored offsprings. 
A

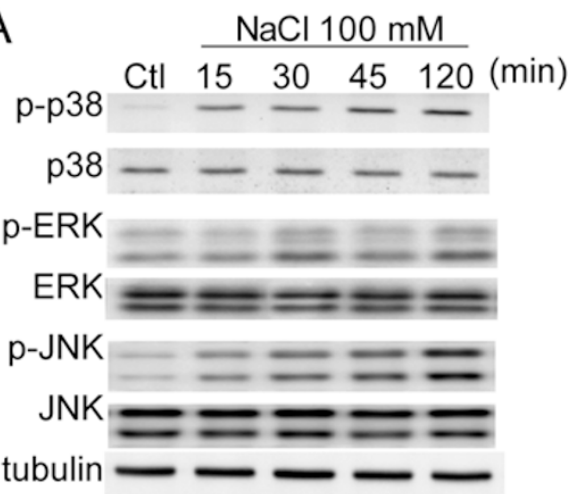

B

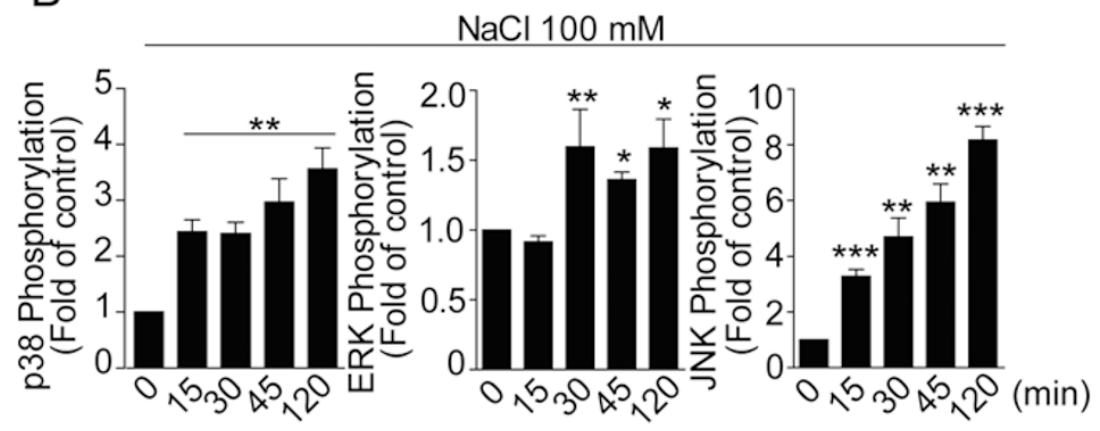

C
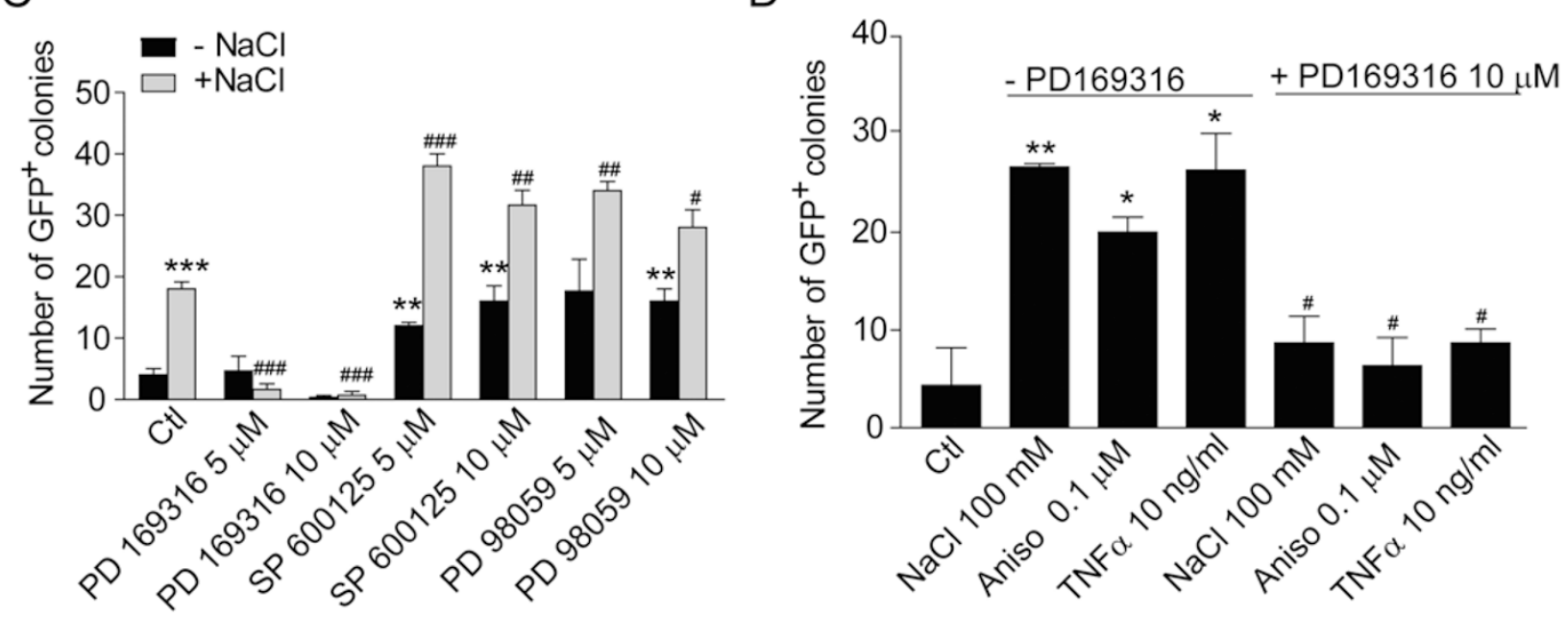

E
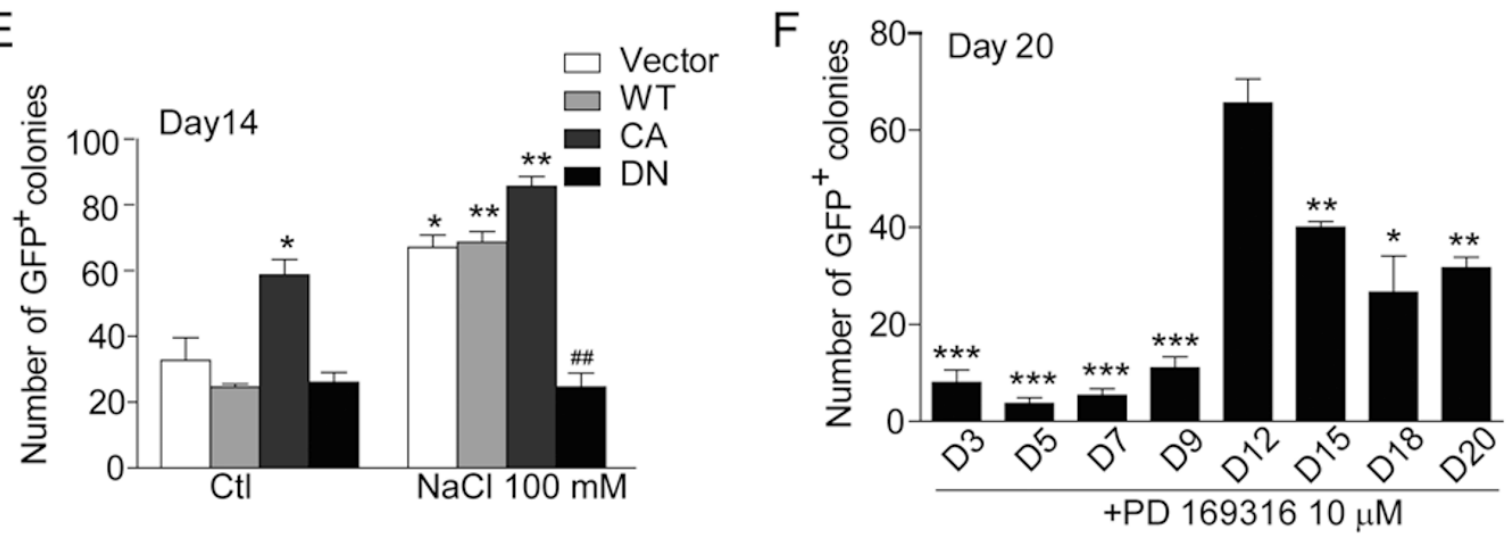

Figure 3 Hyperosmosis promotes reprogramming via p38 activation. (A) Representative western blot of p38, JNK and ERK phosphorylation after $\mathrm{NaCl}$ treatment in MEFs. (B) Statistical analysis of A. Mean \pm SEM of three independent experiments, ${ }^{*} P<0.05,{ }^{* *} P<0.01,{ }^{* * *} P<0.001$. (C) The effect of p38 inhibitor PD169316, JNK inhibitor SP600125 and ERK inhibitor PD98059 on hyperosmosis-enhanced reprogramming in 4F-infected MEFs. Mean \pm SEM of a representative experiment, $n=$ 3 , ${ }^{* *} P<0.01,{ }^{* * *} P<0.001$, versus blank control (first black bar). ${ }^{\#} P<0.05,{ }^{\#} P<0.01,{ }^{\# \#} P<0.001$, versus NaCl control (first gray bar). (D) The effect of anisomycin and TNF $\alpha$ on reprogramming in the presence of PD196316 or not. Mean \pm SEM of a representative experiment, $n=3$. ${ }^{*} P<0.05$, ${ }^{*} P<0.01$, versus control; ${ }^{*} P<0.05$ versus no PD169316 treatment groups. (E) MEFs were infected with 4F and WT-, CA- or DN-p38, and cultured in medium supplemented with $\mathrm{NaCl}_{\text {or not, and GFP }}^{+}$ colonies were counted at day 14 . Mean \pm SEM of a representative experiment, $n=3 .{ }^{*} P<0.05$, ${ }^{* *} P<0.01$, versus vector control without $\mathrm{NaCl} .{ }^{\#} P<0.01$ versus vector control with $\mathrm{NaCl}$. (F) MEFs infected with 4F and CA-p38 were treated with PD169316 from indicated days. GFP ${ }^{+}$colonies were counted at day 20. Mean \pm SEM of a representative experiment, $n=3$. * $P<0.05,{ }^{* *} P<0.01,{ }^{* * *} P<0.001$, versus D12. 
A $=$ OSKM

$\square$ OSKM+NaCl $100 \mathrm{mM}$

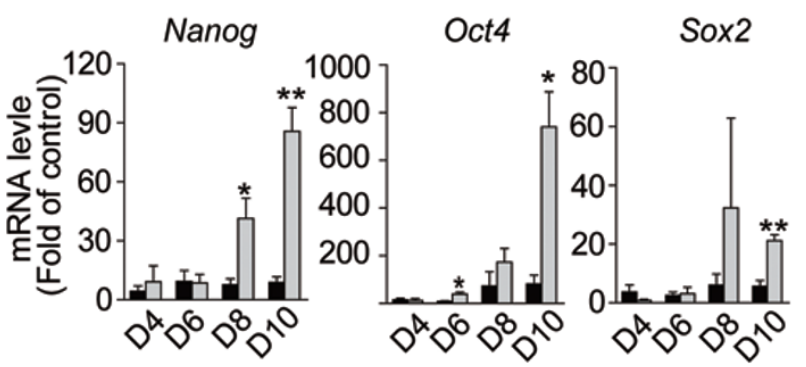

B

- PD169316 $10 \mu \mathrm{M}$
$\square$ +PD169316 $10 \mu \mathrm{M}$
Sox2



C



D $E$

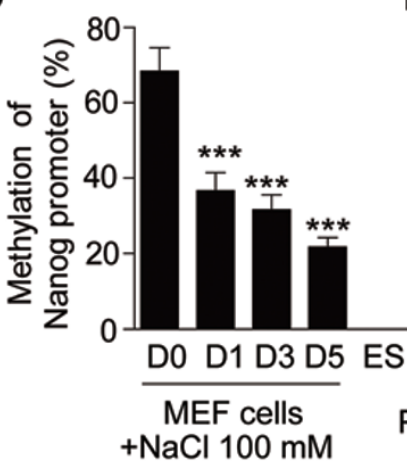

$E$ षิ15 $\quad F$


$\mathrm{NaCl}-+++-$

$\mathrm{AZA}$ - - - + +

PD169316 - $-+{ }_{-}+$



G

$\mathrm{H}$
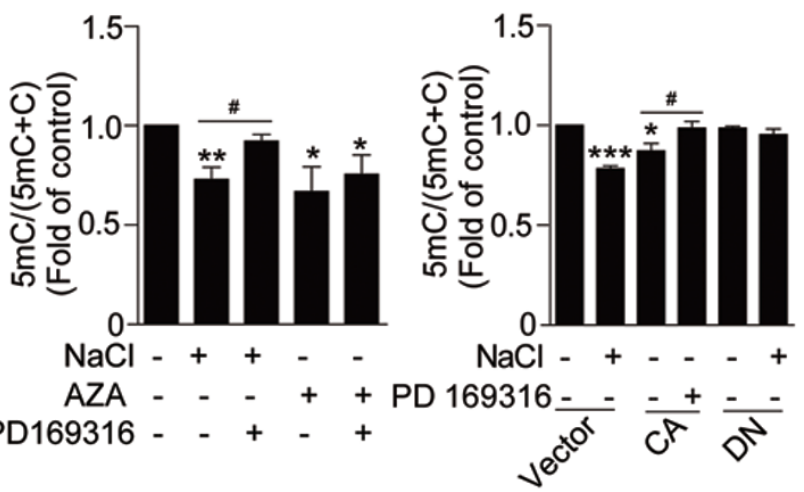

I

iPS cell

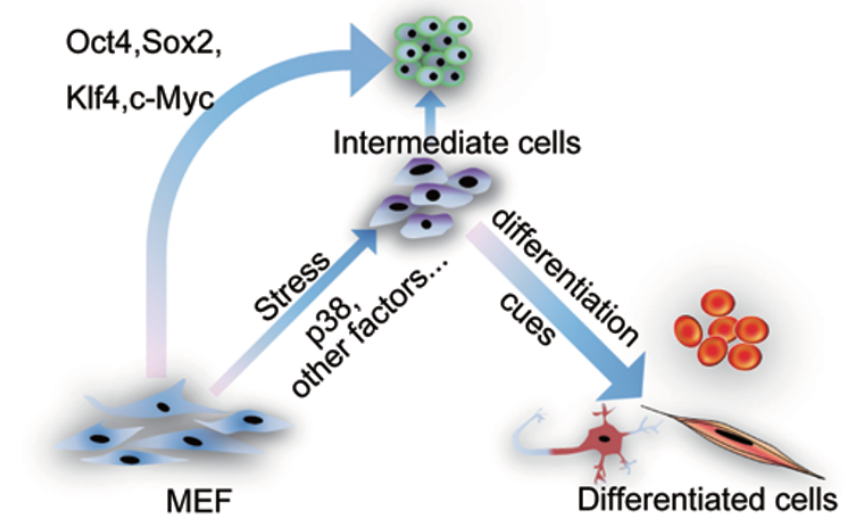

Figure 4 Stress mediated-p38 activation may promote reprogramming by reducing the global DNA methylation level. (A) qPCR analysis of gene expression during 4F-mediated reprogramming in the presence of $\mathrm{NaCl}$ or not. Expression levels were normalized to GAPDH, mean \pm SEM of three independent experiments. ${ }^{*} P<0.05$, ** $P<0.01$, versus no NaCl. (B) Nanog, Oct4 and Sox2 reporter activation by hyperosmosis in HEK293T cells in the presence of PD169316 (10 $\mu$ M) or not. Mean \pm SEM of three independent experiments. ${ }^{*} P<0.05$, ${ }^{* *} P<0.01$, versus no $\mathrm{NaCl}$, no PD169316 control; ${ }^{\# \#} P<0.01$, versus no PD169316 group. (C, D) Bisulfite sequencing analysis of methylation levels at the proximal promoter regions of Oct4 (C) and Nanog (D) in MEFs treated with $\mathrm{NaCl}$ for 1, 3 or 5 days. ES-14 was used as a control. Percent of the CpGs that were methylated are presented. Data are mean \pm SEM of ten clones. ${ }^{* * *} P<0.001$, versus MEF without treatment (D0). (E) Dot blot analysis of 5-mC in DNA extracted from MEFs treated with $\mathrm{NaCl}(100 \mathrm{mM})$ or 5 -Aza $(10 \mu \mathrm{M})$ in the presence of PD169316 $(10 \mu \mathrm{M})$ or not. The levels of $5-\mathrm{mC}$ were normalized to total ssDNA. Mean \pm SEM of six independent experiments. ${ }^{* * *} P<0.001$, versus control; ${ }^{*} P<0.05$. (F) Dot blot analysis of 5-mC in DNA extracted from MEFs infected with WT-, CA- or DN-p38, and treated with $\mathrm{NaCl}(100 \mathrm{mM})$ or PD169316 $(10 \mu \mathrm{M}) .{ }^{* * *} P<0.001$, versus vector; ${ }^{\# \#} P<0.01$. (G) HPLC quantification of genomic content of 5-mC relative to cytosine plus 5-mC in MEFs treated with $\mathrm{NaCl}(100 \mathrm{mM})$ or $5-\mathrm{Aza}(10 \mu \mathrm{M})$ in the presence of PD169316 $(10 \mu \mathrm{M})$ or not. Data are mean \pm SEM of four independent experiments. ${ }^{*} P<0.05$, ${ }^{* *} P<0.01$, versus no treatment; ${ }^{\#} P<0.05$. (H) HPLC quantification of 5-mC in DNA extracted from MEFs infected with WT-, CA- or DNp38, and treated with $\mathrm{NaCl}(100 \mathrm{mM})$ or PD169316 $(10 \mu \mathrm{M}) .{ }^{*} P<0.05,{ }^{* * *} P<0.001$, versus vector control without $\mathrm{NaCl}$ treatment; ${ }^{\#} P<0.05$. (I) A schematic representation of stress-facilitated reprogramming. 
$\mathrm{NaCl}(100 \mathrm{mM})$ treatment significantly reduced the DNA methylation level in MEFs. Similar level of demethylation was also observed in MEFs treated with the DNA methytransferase inhibitor 5-Aza $(10 \mathrm{mM})$. P38 inhibitor PD169316 $(10 \mu \mathrm{M})$ significantly reversed hyperosmosismediated DNA demethylation, though it did not block 5-Aza-induced DNA demethylation (Figure 4E). Interestingly, salt stress has also been reported to alter DNA methylation in certain plants [21]. In MEFs transfected with CA-p 38 , the $5 \mathrm{mC}$ level was also significantly lower compared to the control, and this effect was blocked by PD169316 $(10 \mu \mathrm{M})$. The DN-p38 did not affect the $5 \mathrm{mC}$ level by itself, but it prevented $\mathrm{NaCl}$-induced demethylation of DNA (Figure 4F). HPLC was also used to quantify the $5 \mathrm{mC}$ levels. As shown in Figure $4 \mathrm{G}, \mathrm{NaCl}$ treatment significantly reduced the level of $5 \mathrm{mC}$, and this effect was blocked by PD169316. 5-Aza also induced a similar level of demethylation in the genomic DNA of MEFs, but PD169316 could not block 5-Aza-induced DNA demethylation. Then we measured $5 \mathrm{mC}$ levels in MEF genomic DNA after forced expression of CA-p38 or DN-p38. CA-p38 significantly decreased $5 \mathrm{mC}$ level, and this decrease was blocked by PD169316. DN-p38 had no effect on the methylation level of genomic DNA, but it blocked NaCl-induced demethylation (Figure 4H). Taken together, these data indicate hyperosmotic stress might affect gene transcription by reducing the globel DNA methylation level, and this effect is mediated mainly via the p38 pathway.

\section{Discussion}

Animal development from fertilized, totipotent eggs to individuals is an epigenetically programmed process and was believed to be irreversible in mammals. The groundbreaking work by Yamanaka in 2006 demonstrated that ectopic expression of defined transcription factors could reprogram murine somatic cells to iPS cells [22]. Shortly after that, human iPS cells were generated with similar strategy $[4,23]$. Many iPS cells were extensively characterized and are similar to ES cells in their gene expression patterns and chromatin signatures [24]; some mouse iPS cells can develop into individuals after tetraploid complementation, the most stringent pluripotency test available [25].

Apparently, reprogramming leads to the silencing of somatic genes and reactivation of pluripotency genes concomitant with the resetting of chromatin structure, though the identity of the major chromatin-modifying factors involved in this process remains largely unclear. Higher histone acetylation levels are generally associated with more open chromatin structure and elevated gene expression. Treatment with HDAC inhibitors such as VPA and butyrate results in an enhancement of reprogramming. Methylation at histone $\mathrm{H} 3 \mathrm{~K} 9$ is a repressive chromatin modification that hinders reprogramming. The histone H3K9 methyltransferase G9a inhibitor BIX01294 also enhances reprogramming [9]. DNA methylation is another crucial part of epigenetic regulation. DNA methylation at the fifth position of cytosine has the specific effect of reducing gene expression and plays critical roles in normal cellular differentiation and organismal development [26]. Blocking DNA methylation with DNA methyltransferase inhibitor 5-Aza has also been reported to facilitate iPS cell induction [9]. In this study we demonstrated that environmental stress such as hyperosmosis greatly facilitated iPS cell generation via activation of the p38 pathway. Further study indicates stress-mediated p38 activation might promote reprogramming by reducing the global DNA methylation level and enhancing the expression of pluripotency genes. Recently, hypoxia, another form of environmental stress, has also been reported to facilitate the generation of mouse and human iPS cells [27]. We found hypoxia $\left(5 \%\right.$ and $\left.1 \% \mathrm{O}_{2}\right)$ induced a slight but notable increase ( 1.5 -fold) in p38 phosphorylation (Supplementary information, Figure S4G and $\mathrm{S} 4 \mathrm{H})$. We speculate that $\mathrm{p} 38$ activation might play a role, but it is not likely to be the main mechanism of hypoxiaenhanced reprogramming. Other mechanisms, such as Hif-2 $\alpha$-mediated Oct4 transcription [28], might play a more important role.

Dramatic change in gene expression is a major component of stress responses, and such change is achieved by the modulation of chromatin structure, transcription, mRNA stability and translation via intracellular signaling. Environmental stresses, such as osmostress, UV, heat stress, oxidative stress, heavy metals and chemicals, as well as physiological mediators, such as inflammatory cytokines, activate the highly conserved stressactivated protein kinases (SAPKs) pathways [29]. The p38 MAPKs are the mammalian orthologues of yeast SAPK Hog1, an osmo-sensing protein. Stresses with various sensing mechanisms converge through different MAP3Ks on $\mathrm{p} 38$, which in turn regulate the activity of downstream transcription factors and regulators of chromatin remodeling [3]. Hyperosmotic stress activates other signaling pathways in addition to $\mathrm{p} 38$. We also found hyperosmosis activated all three MAPK pathways, p38, JNK and ERK. It is interesting to note that only p38 activation contributed to the positive effect of hyperosmosis on reprogramming. In fact, the activation of the other two pathways was detrimental [15] to iPS cell induction even though the summation of the signaling under hyperosmosis led to a beneficial effect. The duration of $\mathrm{p} 38$ activation was also critical to the enhancement effect, as 
prolonged treatment with hyperosmotic condition or constant activation of p38 eventually leads to less iPS cells. This is understandable since cells can quantify stress and activate a death program when tolerance limits are exceeded [30].

Enhancement of reprogramming via p38 activation was surprising since p38 activation had long been associated with proliferation inhibition and differentiation induction. P38 activation also leads to p53-mediated premature senescence [31], which has been reported to be the major roadblock of somatic cell reprogramming [32]. Nevertheless, we observed that hyperosmosis-induced p38 activation stimulated the expression of pluripotency genes with or without the treatment of Yamanaka factors. Several pluripotency genes, such as Sox 2 and $M y c$, have also been found to be upregulated in MEFs in a whole genome analysis of p38-mediated gene expression upon stress [33]. Thus we speculate that a more general mechanism, rather than the expression of specific genes, might underlie the effect of stress-activated p38 on reprogramming. We found stress-mediated p38 activation led to a reduction of global DNA methylation level similar to the treatment of 5-Aza, and rendered the cells to enter an epigenetically unstable intermediate state. With the presence of Yamanaka factors or various differentiation cues, these cells are then more readily to be reprogrammed into the pluripotent state or induced to differentiate (Figure 4I).

In summary, we found stress-mediated p38 activation led to a reduction of global DNA methylation level, and rendered the cells to enter an epigenetically unstable intermediate state, resulting in a higher reprogramming efficiency. Our data not only provide a simple and safe way to facilitate the generation of iPS cells, but also demonstrate how simple environmental stress like hyperosmosis helps to alter the fate of cells via intracellular signaling and epigenetic modulation.

\section{Materials and Methods}

\section{Derivation of MEFs and cell culture}

OG2 mice, which carry a transgenic Oct4 promoter driven GFP reporter, were mated with $\mathrm{C} 57$ mice and MEF cells were isolated from E12.5 embryos heterozygous for the Oct4-GFP transgenic allele. Gonads and internal organs were removed before processing for isolation of MEF cells. MEFs were grown in DMEM supplemented with 10\% FBS, $2 \mathrm{mM}$ L-glutamax, $0.1 \mathrm{mM}$ nonessential amino acids (NEAA), 100 units $/ \mathrm{ml}$ penicillin and $100 \mu \mathrm{g} / \mathrm{ml}$ streptomycin. Isolated MEF cells in early passages (up to passage 3) were used for further experiments.

\section{Mouse iPS cell generation}

Retrovirus were produced by transfection of plat-E cells with pMXs retroviral vectors containing the coding sequences of mouse
Oct4, Sox 2, Klf4 and c-Myc (obtained from Addgene). MEFs (passage 1-3) were seeded at a density of 150000 cells per well in sixwell plate $18 \mathrm{~h}$ before infection. Virus containing supernatants, supplemented with $4 \mu \mathrm{g} / \mathrm{ml}$ polybrene, were added onto the plates of MEF cell cultures and spined at $2500 \mathrm{rpm}$ for $90 \mathrm{~min}$ to ensure their infection. Medium was changed immediately after virus transduction and this day is counted as 'Day 0'. Two days post virus infection, MEFs were trypsinized into single cells and reseeded at a density of 5000 cells per well on 96-well plates or 7500 cells per well on 24-well plates pre-seeded with irradiated MEF feeders, supplemented with mES medium (DMEM supplemented with 15\% FBS, 2 mM L-glutamax, $0.1 \mathrm{mM}$ NEAA, $0.1 \mathrm{mM}$ $\beta$-mercaptoethanol $1000 \mathrm{U} / \mathrm{ml} \mathrm{LIF,} 100 \mathrm{units} / \mathrm{ml}$ penicillin and $100 \mu \mathrm{g} / \mathrm{ml}$ streptomycin). At day 6 , culture medium was replaced with KSR medium (knockout-DMEM supplemented with $15 \%$ knockout serum replacement, $2 \mathrm{mM}$ L-glutamax, $0.1 \mathrm{mM}$ nonessential amino acids (NEAA), $0.1 \mathrm{mM} \beta$-mercaptoethanol, $1000 \mathrm{U} /$ $\mathrm{ml} \mathrm{LIF,} 100$ units/ml penicillin and $100 \mu \mathrm{g} / \mathrm{ml}$ streptomycin). $\mathrm{NaCl}$ or other chemicals were added from day 3 with various durations.

$\mathrm{GFP}^{+}$colonies were scanned and counted using Acumen eX3 microplate scanner (TTP Labtech) or ImageXpress ${ }^{\mathbb{B}}$ Micro System (Moleculer Devices) from day 8 post infection. GFP $^{+}$colonies were also trypsinized and then analyzed using a FACS Calibur (BD). $\mathrm{GFP}^{+}$cells were gated with a control signal from the PE channel and a minimum of 10000 events were recorded.

\section{Alkaline phosphatase and immunofluorescent staining}

For alkaline phosphatase staining, iPS cells were fixed with $4 \%$ paraformaldehyde (PFA) in PBS for 45 seconds, rinsed once with PBS and detection was performed using a leukocyte alkaline phosphatase kit (Sigma, catalog No 85L3R) according to the manufacturer's protocol. For immunofluorescent staining, cells were fixed with $4 \%$ PFA and incubated with primary antibodies against mSSEA-1 (Santa Cruz, sc-21702) and mNanog (Millipore, AB5731), followed by the appropriate secondary antibodies conjugated to Alexa Fluor 555 (Invitrogen). Nuclei were counterstained with Hoechst 33342 (Sigma). Images were taken with an Olympus IX51 inverted fluorescent microscope or an Olympus FV10i confocal microscope.

\section{Real-time PCR}

Total mRNA was isolated using TRIzol (Invitrogen) and $2 \mu \mathrm{g}$ RNA were used to synthesize cDNA using PrimeScriptTM RT reagent kit (Takara, DRR037A) according to the manufacturer's protocol. Real-time PCR was performed using JumpStartTM TaqReady MixTM (Sigma, D7440) with Eva Green (Biotium) and analyzed with a Stratagene Mx 3000P thermal cycler. For semiquantitative PCR analysis, the cDNA solution was amplified for 45 cycles at an optimal annealing temperature. Primers sequences are supplied in Supplementary information, Table S1.

\section{Bisulfite sequencing}

Bisulfite sequencing was used to detect DNA methylation in the promoter regions of Oct4 and Nanog as described [22]. In brief, genomic DNA was isolated and bisulfite conversion was performed in agarose beads as described [34]. Nested PCR was performed to amplify the promoter regions after bisulfite conversion and the PCR products were cloned into pMD19-T vectors (Takara). Ten randomly selected clones from each sample were sequenced 
and analyzed. PCR primers are listed in Supplementary information, Table S2.

\section{Western blot}

Cells were lysed, sonicated and boiled at $95-100{ }^{\circ} \mathrm{C}$ for $5 \mathrm{~min}$ in sample buffer $(50 \mathrm{mM}$ Tris- $\mathrm{HCl}, 2 \% \mathrm{w} / \mathrm{v}$ SDS, $10 \%$ glycerol, $1 \% \beta$-mercaptoethanol, $0.01 \%$ bromophenyl blue ( $\mathrm{pH}$ 6.8)). Cell lysates were separated on SDS-PAGE and transferred to polyvinylidene difluoride membranes. The membranes were first incubated with blocking buffer (TBS with $0.05 \%$ Tween $20,10 \%$ nonfat milk) for $1 \mathrm{~h}$ at room temperature and then incubated overnight at $4{ }^{\circ} \mathrm{C}$ in buffer containing rabbit anti- $\alpha$-Tubulin $(11 \mathrm{H} 10)$ (1:10 000; CST 2125), rabbit anti-p38 (1:1 000; CST 9212), rabbit anti-p-p38(1:1 000; CST 9211), rabbit anti-JNK (1:1 000; CST 9258), rabbit anti-p-JNK(1:1 000; CST 4668), rabbit anti-ERK (1:1 000; CST 9102), rabbit anti-p-ERK(1:1 000; CST 4370), or rabbit anti-p-MK2 (1:1 000; CST 3316). The membranes were washed thrice and incubated with goat anti-rabbit IgG HRP (1:10 000) or goat anti-mouse IgG HRP (1:10 000) for $1 \mathrm{~h}$. After washing, immunostaining was visualized using Western Lightning Ultra (Perkin Elmer) and ChemiDoc imaging system (Bio-Rad).

\section{Teratoma formation, chimera production and germline} transmission

About $1 \times 10^{6}$ iPS cells were suspended in $200 \mu 1 \mathrm{mES}$ medium and injected into NOD-SCID mice to form teratomas. Four weeks after injection, teratomas were harvested, fixed overnight with 4\% PFA, embedded in paraffin, sectioned, hematoxylin and eosin stained and analyzed. For production of chimeric mice, zygotes were isolated from superovulated female ICR mice and iPS cells were injected into the resulting blastocysts. Chimeras were produced by implantation of injected blastocysts into the pseudopregnant ICR mice. The chimeras were crossed with ICR mice to confirm the germline transmission.

\section{Luciferase reporter assays}

For Nanog, Oct4 and Sox 2 reporter activitiy, plasmids encoding promoter regions of Nanog (50 ng), Oct4 (100 ng) or Sox2 (25 ng) together with a firefly luciferase reporter were cotransfected with 25 ng Renilla luciferase driven by TK-promoter (Promega) into HEK293T cells seeded on 96-well plate at a density of 40000 per well using Fugene HD (Roche). Chemicals were added $18 \mathrm{~h}$ after transfection and firefly and renilla luciferase activities were measured $24 \mathrm{~h}$ after drug treatment with the Dual-Glo Luciferase Assay System (Promega) using an EnVision multilabel plate reader (Perkin Elmer).

\section{Dots blot analysis of total DNA 5-mC}

MEFs were incubated in medium supplemented with $100 \mathrm{mM}$ $\mathrm{NaCl}$ for $72 \mathrm{~h}$. Genomic DNA was isolated using the DNA Isolation Kit (Promega) according to the manufacturer's instructions and 200 ng DNA from each sample was dotted onto the nitrocellulose membrane (GE Amersham). Membrane was exposed to UV light for $5 \mathrm{~min}$ and then incubated in blocking buffer for $1 \mathrm{~h}$ at room temparature. Membrane was incubated overnight with the 5-mC antibody (Active motif 39649) in blocking buffer at $4{ }^{\circ} \mathrm{C}$. After through washing, membrane was probed with HRP-conjugated secondary antibody and visualized using Western Lightning Ultra and ChemiDoc imaging system (Bio-Rad).

\section{HPLC quantification of total DNA 5-mC}

The genomic DNA was extracted by phenol/chloroform then treated with RNase A/T1 twice (50 $\mu \mathrm{g}$ RNase A and $1000 \mathrm{U}$ RNase T1 per $100 \mu \mathrm{g}$ DNA), at least $4 \mathrm{~h}$ each time. The DNA was purified by phenol/chloroform, precipitated in 70\% ethanol, and dissolved in water. Approximately, $40 \mu \mathrm{g}$ DNA was hydrolyzed with $0.1 \mathrm{U}$ of nuclease $\mathrm{P} 1$ (Sigma) at $50{ }^{\circ} \mathrm{C}$ for at least $1 \mathrm{~h}$ or at $37{ }^{\circ} \mathrm{C}$ overnight (the reaction volume is $80 \mu \mathrm{l}$, containing $20 \mathrm{mM}$ $\mathrm{NaOAc}, \mathrm{pH} 5.3$ and $0.2 \mathrm{mM} \mathrm{ZnSO}_{4}$ ). $8 \mu \mathrm{l}$ of $10 \times$ CIAP buffer and $1 \mu \mathrm{l}$ of calf intestinal alkaline phosphatase (TaKaRa) were then added to the reaction followed by incubation for at least $2 \mathrm{~h}$ or overnight at $37{ }^{\circ} \mathrm{C}$. The reaction products were then analyzed on an Agilent 1200 HPLC instrument with an AQ-C18 column (5$\mu \mathrm{m}$ particle size, $25 \mathrm{~cm} \times 4.6 \mathrm{~mm}$ ). The mobile phase was $10 \mathrm{mM}$ $\mathrm{KH}_{2} \mathrm{PO}_{4}, \mathrm{pH} 3.7$, running at $1 \mathrm{ml}$ per min, and the detector was set at $280 \mathrm{~nm}$. Standard compounds 2'-deoxycytidine and 5-methyl 2 '-deoxycytidine were bought from Sigma and TCI.

\section{Acknowledgments}

We thank Qiuhong Hua for her technical assistance in iPS cell assay development. We thank Drs Min Gu and Yufei He for their technical assistance in HPLC operation. We thank professors David Engelberg and Helmut Holtmann for providing the original plasmids of p38 mutants. This project was supported by grants from the Chinese Academy of Sciences (XDA01040301), Ministry of Science and Technology of China (2009CB940900, 2010CB944900, 2011CB965104, 2011DFB30010) and Shanghai Commission of Science and Technology (08DZ2291300, 09DZ2291200).

\section{References}

1 Hochachka PW, Somero GN, eds. Biochemical adaptation: Mechanism and process in physiological evolution. Oxford University Press, USA, 2002.

2 Charmandari E, Tsigos C, Chrousos G. Endocrinology of the stress response. Annu Rev Physiol 2005; 67:259-284.

3 de Nadal E, Ammerer G, Posas F. Controlling gene expression in response to stress. Nat Rev Genet 2011; 12:833-845.

4 Takahashi K, Tanabe K, Ohnuki M, et al. Induction of pluripotent stem cells from adult human fibroblasts by defined factors. Cell 2007; 131:861-872.

5 Mattout A, Biran A, Meshorer E. Global epigenetic changes during somatic cell reprogramming to iPS cells. $J$ Mol Cell Biol 2011; 3:341-350.

6 Kim JB, Sebastiano V, Wu GM, et al. Oct4-induced pluripotency in adult neural stem cells. Cell 2009; 136:411-419.

7 Stadtfeld M, Nagaya M, Utikal J, Weir G, Hochedlinger K. Induced pluripotent stem cells generated without viral integration. Science 2008; 322:945-949.

8 Zhou HY, Wu SL, Joo JY, et al. Generation of induced pluripotent stem cells using recombinant proteins. Cell Stem Cell 2009; 4:381-384.

9 Feng B, Ng JH, Heng JCD, Ng HH. Molecules that promote or enhance reprogramming of somatic cells to induced pluripotent stem cells. Cell Stem Cell 2009; 4:301-312.

10 Kultz D, Burg M. Evolution of osmotic stress signaling via 
MAP kinase cascades. J Exp Biol 1998; 201:3015-3021.

11 Shi Y, Desponts C, Do JT, Hahm HS, Scholer HR, Ding S. Induction of pluripotent stem cells from mouse embryonic fibroblasts by Oct 4 and Klf4 with small-molecule compounds. Cell Stem Cell 2008; 3:568-574.

12 Skjerdal OT, Sletta H, Flenstad SG, Josefsen KD, Levine DW, Ellingsen TE. Changes in intracellular composition in response to hyperosmotic stress of $\mathrm{NaCl}$, sucrose or glutamic acid in Brevibacterium lactofermentum and Corynebacterium glutamicum. Appl Microbiol Biot 1996; 44:635-642.

13 Melton DA, Huangfu DW, Maehr R, et al. Induction of pluripotent stem cells by defined factors is greatly improved by small-molecule compounds. Nat Biotechnol 2008; 26:795-797.

14 Wang Q, Xu X, Li J, et al. Lithium, an anti-psychotic drug, greatly enhances the generation of induced pluripotent stem cells. Cell Res 2011; 21:1424-1435.

15 Silva J, Barrandon O, Nichols J, Kawaguchi J, Theunissen TW, Smith A. Promotion of reprogramming to ground state pluripotency by signal inhibition. Plos Biol 2008; 6:2237-2247.

16 Diskin R, Askari N, Capone R, Engelberg D, Livnah O. Active mutants of the human $\mathrm{p} 38$ alpha mitogen-activated protein kinase. J Biol Chem 2004; 279:47040-47049.

17 Winzen R, Kracht M, Ritter B, et al. The p38 MAP kinase pathway signals for cytokine-induced mRNA stabilization via MAP kinase-activated protein kinase 2 and an AU-rich regiontargeted mechanism. EMBO J 1999; 18:4969-4980.

18 Mammone T, Ingrassia M, Goyarts E. Osmotic stress induces terminal differentiation in cultured normal human epidermal keratinocytes. In Vitro Cell Dev Biol Anim 2008; 44:135-139.

19 Al-Khalili L, Chibalin AV, Yu M, et al. MEF2 activation in differentiated primary human skeletal muscle cultures requires coordinated involvement of parallel pathways. Am J Physiol Cell Physiol 2004; 286:C1410-C1416.

20 Li YQ, Jiang BH, Ensign WY, Vogt PK, Han JH. Myogenic differentiation requires signalling through both phosphatidylinositol 3-kinase and p38 MAP kinase. Cell Signal 2000; 12:751-757.

21 Verhoeven KJF, Jansen JJ, van Dijk PJ, Biere A. Stressinduced DNA methylation changes and their heritability in asexual dandelions. New Phytol 2010; 185:1108-1118.

22 Takahashi K, Yamanaka S. Induction of pluripotent stem cells from mouse embryonic and adult fibroblast cultures by defined factors. Cell 2006; 126:663-676.

23 Yu JY, Vodyanik MA, Smuga-Otto K, et al. Induced pluripotent stem cell lines derived from human somatic cells. Science 2007; 318:1917-1920.

24 Maherali N, Sridharan R, Xie W, et al. Directly reprogrammed fibroblasts show global epigenetic remodeling and widespread tissue contribution. Cell Stem Cell 2007; 1:55-70.

25 Kang L, Wang JL, Zhang Y, Kou ZH, Gao SR. iPS Cells can support full-term development of tetraploid blastocyst-complemented embryos. Cell Stem Cell 2009; 5:135-138.

26 Cooper DN. Eukaryotic DNA Methylation. Hum Genet 1983; 64:315-333.

27 Yoshida Y, Takahashi K, Okita K, Ichisaka T, Yamanaka S. Hypoxia enhances the generation of induced pluripotent stem cells. Cell Stem Cell 2009; 5:237-241.

28 Covello KL, Kehler J, Yu HW, et al. HIF-2 alpha regulates Oct-4: effects of hypoxia on stem cell function, embryonic development, and tumor growth. Gene Dev 2006; 20:557-570.

29 Zarubin T, Han JH. Activation and signaling of the p38 MAP kinase pathway. Cell Res 2005; 15:11-18.

30 Kultz D. Molecular and evolutionary basis of the cellular stress response. Annu Rev Physiol 2005; 67:225-257.

31 Han J, Sun P. The pathways to tumor suppression via route p38. Trends Biochem Sci 2007; 32:364-371.

32 Marion RM, Strati K, Li H, et al. A p53-mediated DNA damage response limits reprogramming to ensure iPS cell genomic integrity. Nature 2009; 460:1149-1153.

33 Ferreiro I, Joaquin M, Islam A, et al. Whole genome analysis of 38 SAPK-mediated gene expression upon stress. BMC Genomics 2010; 11:144.

34 Hajkova P, el-Maarri O, Engemann S, Oswald J, Olek A, Walter J. DNA-methylation analysis by the bisulfite-assisted genomic sequencing method. Methods Mol Biol 2002; 200:143154.

(Supplementary information is linked to the online version of the paper on the Cell Research website.)

(a) This work is licensed under the Creative Commons Attribution-NonCommercial-No Derivative Works 3.0 Unported License. To view a copy of this license, visit http:// creativecommons.org/licenses/by-nc-nd/3.0 